\title{
Genetic and environmental factors predict multivariate trajectories of maternal distress after birth
}

\section{Authors}

Eva Unternaehrer ${ }^{* 1,2,3}$, Keelin Greenlaw ${ }^{* 4,5}$, Antonio Ciampi ${ }^{4}$, Lawrence M Chen ${ }^{1}$, Andree-

Anne Bouvette-Turcot ${ }^{1}$, Shantala Hari Dass ${ }^{1}$, Irina Pokhvisneva ${ }^{1}$, Patricia P Silveira ${ }^{1}$, Katherine

T Cost $^{6}$, Helene Gaudreau ${ }^{1}$, Elika Garg ${ }^{1}$, Yuecai Zhu ${ }^{1}$, Thao TT Nguyen ${ }^{1}$, Marie Forest ${ }^{4}$, Nelson

Yao $^{1}$, Julie L MacIsaac ${ }^{7}$, Lisa M McEwen ${ }^{7}$, Michael S Kobor ${ }^{7}$, Alison S Fleming ${ }^{8}$, Meir S

Steiner ${ }^{9}$, John E Lydon ${ }^{10}$, Robert Levitan ${ }^{11}$, Michael J Meaney ${ }^{1,12,13}$, Kieran J O’Donnell ${ }^{1,13,14}$,

Celia MT Greenwood ${ }^{4}$, and the MAVAN research team

*Equally contributing first authors

\section{Affiliations}

${ }^{1}$ Douglas Mental Health University Institute and Ludmer Centre for Neuroinformatics and Mental Health, McGill University, Montreal, Canada; ${ }^{2}$ Child- and Adolescent Research Department, Psychiatric University Hospitals Basel (UPK), University of Basel, Switzerland; ${ }^{3}$ Department of Psychology, University of Konstanz, Konstanz, Germany; ${ }^{4}$ Lady Davis Institute for Medical Research, Jewish General Hospital, Montreal, Canada; ${ }^{5}$ Department of Psychology, Concordia University, Montreal, Canada; ${ }^{6}$ The Hospital for Sick Children, Toronto, Canada; ${ }^{7} \mathrm{BC}$ Children's Hospital Research Institute, University of British Columbia, Vancouver, Canada; ${ }^{8}$ University of Toronto Mississauga, Toronto, Canada; ${ }^{9}$ McMaster University, Hamilton, Canada; ${ }^{10}$ McGill University, Montreal, Canada; ${ }^{11}$ Centre for Addiction and Mental Health, Toronto, Canada; ${ }^{12}$ Singapore Institute for Clinical Sciences, Singapore; ${ }^{13}$ Child and Brain Development Program, CIFAR, Toronto, Canada; ${ }^{14}$ Yale Child Study Center, \& Department of Obstetrics, Gynecology and Reproductive Sciences, Yale University, New Haven, Connecticut, USA

Corresponding Author: Eva Unternaehrer (eva.unternaehrer@unibas.ch)

Keywords: general stress, latent class modeling, marital stress, parenting stress, postpartum distress, symptoms of depression

Please note that this manuscript is a pre-print and has not been peer-reviewed. 


\begin{abstract}
Background: Maternal distress influences her own wellbeing and shapes her offspring's psychosocial adjustment and neurodevelopment across childhood. The aim of this study was to analyze multivariate trajectories of maternal postpartum distress using a latent class modeling approach and to find genetic and psychosocial factors that predict membership within a given group (latent class).

Methods: Maternal self-reports of depressive symptoms, parenting stress, general stress, and marital stress were measured at regular intervals during the first six years postpartum in 261 mothers participating in the Maternal Adversity, Vulnerability and Neurodevelopment Study. Genetic risk was determined by calculating a polygenic risk score for Major Depressive Disorder (MDD-PRS). Additionally, we assessed maternal history of early life adversity (mELA), educational level, and prenatal symptoms of depression as psychosocial risk factors. Using Latent Gold® Software, we identified latent classes of mothers based on their 1) average levels of distress and 2) change in distress over time.

Results: We identified four latent classes based on average levels of distress and found that class membership probability was influenced by an interaction between MDD-PRS and prenatal depressive symptoms (Wald $\operatorname{Interaction}(3)=13.19, \mathrm{p}=0.004 ; \operatorname{Wald}_{\mathrm{MDD}-\operatorname{PRS}}(3)=6.02, p=0.11$; Wald Depression $\left._{(3)=41.96 ;} p<0.001\right), \operatorname{mELA}($ Wald $(3)=8.64, p=0.035)$, and educational level (Wald $(3)=11.61, p=0.009)$. Furthermore, we found five classes of mothers with distinct acrosstime trajectories, which were associated with mELA (Wald(3)=12.67, $p=0.013)$. Conclusions: Our findings might become relevant in the clinical setting, e.g. for identifying pregnant women at risk for distress in the postpartum based on her prenatal symptoms of depression and genetic risk, mELA, and educational level.
\end{abstract}




\section{Introduction}

Maternal emotional wellbeing is an important concern for both women's health as well as the growth and development of their offspring $(1,2)$. Although maternal mood commonly improves in the postpartum phase $(3,4)$, onset of mood disturbances during pregnancy or the early postpartum, such as perinatal depression, are estimated to affect $10-20 \%$ of mothers in developed countries (4). Moreover, an even higher percentage of mothers experience subclinical levels of depressive symptoms that are sufficient to compromise psychosocial function and parenting (5). These mood disturbances are heterogeneous in their onset, course, and symptomatology (6).

In addition, maternal mood disturbances, such as symptoms of depression, might only be one aspect of maternal emotional wellbeing during pregnancy and the postpartum with impact on child development $(7,8)$. More severe symptoms of depression are commonly associated with increased levels of distress in different areas of everyday life, including stress related to parenting $(9,10)$, to the relationship with the romantic partner $(11)$ or general daily life stress. However, it has not been investigated whether the trajectories in these multiple aspects of maternal emotional wellbeing parallel each other or show distinct patterns of change. Furthermore, postpartum emotional wellbeing may be influenced by multiple factors, including genetic predispositions and psychosocial factors, such as childhood adversities, mental disorders existing pre-conception, and/or socioeconomic status $(3,11,12)$. While studies on psychosocial risk factors have commonly yielded consistent results, previous findings on a genetic contribution toward peripartum depression are mixed (13). However, a recent study found that the MDD-PRS predicted prenatal and postnatal depressive symptoms, albeit the latter prediction was mediated through prenatal depressive symptoms (14). In addition, latent profile analysis of 
14 prenatal and two postnatal ( 2 weeks after delivery, 6 months after delivery) depressive symptoms scores yielded a consistently low, moderate, and high subgroup. Likewise, a higher PRS predicted greater odds to belong to the consistently moderate or high latent profile groups (14).

The aim of the current study was to analyze multivariate trajectories of maternal distress using a latent class modeling approach. A latent class (LC) is a categorical variable, which distinguishes classes of mothers with similar trajectories in multiple measures of distress. This approach allows us to examine trajectories in multiple variables simultaneously while taking into account individual-level variation in symptom trajectories accounting for relevant covariates and their interaction on these trajectories. Previous studies applying latent class or growth-curve modeling approaches to study trajectories in postpartum emotional wellbeing, mostly in postpartum symptoms of depression, found that the overall level of depressive symptom severity, rather than the change over time, determined the trajectory groups (for a systematic review see 6). Moreover, while research on postpartum depression has examined genetic and psychosocial risk factors, their association and interaction with heterogeneous profiles encompassing multiple aspects of emotional wellbeing is unknown. In the current study, we decomposed each distress trajectory into two components to cover both aspects, an average level of distress across timepoints (Average Distress Model, ADM) and the actual change over time - i.e. a mother's distress score at a respective time point in relation to her average distress across time - (Centered Trajectory Model, CTM). We hypothesized that the latent class structure and its relationships with potential genetic and psychosocial risk factors would differ between average levels of distress and changes in distress over time. 
The data for this study was derived from the Maternal Adversity, Vulnerability and Neurodevelopment (MAVAN) project, a community cohort study following mother-child dyads from pregnancy to when the child is twelve years old. We focused on four dimensions of maternal distress: depressive symptoms, general daily life stress, parenting stress, and stress in the relationship with the spouse (marital stress), measured at regular intervals over the first six years after birth. The factors examined in this study consisted of a PRS (15) for major depressive disorder (MDD-PRS) (16) - and three psychosocial factors related to postpartum wellbeing: maternal history of early life adversity (mELA) (17-19), levels of maternal education (12), and prenatal symptoms of depression (20). To expand on previous research, we also examined a potential interaction between the MDD-PRS and psychosocial factors considered in previous studies on general depression $(21,22)$, but not in postpartum mood (23).

\section{Methods and Materials}

\section{Participants and Procedure}

The study sample was a subsample ( $n=261)$ of the 496 families originally participating in the MAVAN project. Pregnant women were recruited during the second trimester of pregnancy in Montreal and Hamilton, Canada, between 2003 and 2005; follow-ups occurred at 6, 12, 24, 36, 48, 60 and 72 months after birth. For additional information on recruitment and the MAVAN study design see (24). All mothers provided written informed consent at the time of study enrollment. The protocol was approved by the Research Ethics Board at the Douglas Mental Health University Institute (Montreal cohort) and St-Joseph's Hospital (Hamilton cohort). Our final sample consisted of 261 mothers (age at time of childbirth: $M=30.60$ years; $S D=4.88$ 
years, range: 18 to 43 years) who had (i) measurements at a minimum of three time points $(n=375)$, and (ii) available genotyping data $(n=264)$. The study sample included 10 mothers without partners and thus without observed measures of marital stress; the sensitivity of the results was evaluated by repeating all analyses excluding these 10 mothers. Participant's Characteristics are provided in Table 1.

Table 1

Participant characteristics

\begin{tabular}{lcc}
\hline & $\mathrm{N}$ & $\%$ \\
\hline Recruitment Site & 143 & 54.79 \\
$\quad$ Montréal & 118 & 45.21 \\
$\quad$ Hamilton & & \\
Offspring Gender & 135 & 51.72 \\
$\quad$ Male & 126 & 48.28 \\
$\quad$ Female & 54 & 20.69 \\
Income Category ${ }^{1}$ & 170 & 65.13 \\
$\quad$ Low Income & 37 & 14.18 \\
$\quad$ Average/High Income & 261 & \\
$\quad$ Information Missing & 19 & 7.28 \\
Level of Education & 19 & 7.28 \\
$\quad$ High School Diploma or Less & 85 & 32.57 \\
$\quad$ Attended Community College & 126 & 48.28 \\
$\quad$ Completed Community College & 12 & 4.60 \\
$\quad$ University Degree & & \\
$\quad$ Information Missing &
\end{tabular}

\section{Outcome Variables: Measures of Distress}

After delivery, mothers completed questionnaires on symptoms of depression (Centre of Epidemiological Studies Depression Scale, CES-D (25)), parenting stress (Parental Stress Index, PSI (26)), stress in the relationship with the spouse (referred to as marital stress, although not all mothers were married to their partners, Marital Stress Index, MSI (27)), and general perceived 
stress (Perceived Stress Scale, PSS (28)) at 6, 12, 24, 36, 48, 60, and 72 months postpartum.

Higher scores on these instruments indicate higher levels of distress. Detailed information on the applied questionnaires can be found in the Supplementary Materials (S1).

\section{Covariates}

Investigated covariates consisted of (i) psychosocial factors: mELA, prenatal CES-D score, and maternal level of education, and (ii) a genetic factor: the MDD-PRS $(15,16)$.

Psychosocial factors. mELA was assessed using a combined measure of the Parental Bonding Instrument (PBI) (29) 'maternal care' subscale and the five subscales of the Childhood Trauma Questionnaire (CTQ) (30): 'physical neglect', 'emotional neglect', 'physical abuse', 'emotional abuse', and 'sexual abuse'. The six measures were summarized into a composite score of mELA using principal component analysis (PCA) and retaining the first principal component (as described previously in 31 ). This first principal component captured $58.41 \%$ of the total variation observed in the six mELA measures. A high score indicates high levels of adversity. Prenatal depressive symptoms were measured using the CES-D total score assessed in the third pregnancy trimester. Due to the high proportion of mothers with a university degree in our sample (Table 1), we summarized the maternal educational level with two groups: those with $(n=126)$ and those without $(n=123)$ a university degree.

Genetic data. Genetic risk was assessed using a polygenic risk score for depression. Genetic Material was collected and extracted from maternal saliva samples using Oragene DNA Collection Kits OG-500 (DNA Genotek Inc., Ottawa, Canada). Genotypes were described using the Illumina PsychChip and PsychArray (Illumina Inc., Victoria, Canada). Data on quality 
control (PLINK 1.9, www.cog-genomics.org/plink/1.9/) (32), and missing genotype imputation (Wellcome Trust Sanger Institute, Hinxton, UK(33)), is provided in the Supplementary Materials (S2).

\section{Data Preparation}

Polygenic risk score. A polygenic-risk score (PRS) is an aggregate score of risk-associated single nucleotide polymorphisms (SNPs) across the genome. As such, MDD-PRS represents the genetic risk for MDD from additive effects of common genetic variants (34). Here, MDD-PRS was calculated as the summation of risk allele counts multiplied by their effect size (log odds ratio) based on the summary statistics provided by the Psychiatric Genomics Consortium's GenomeWide Association Study (GWAS) for MDD (16); for more detailed information see Supplementary Materials (S2) and (35). SNP inclusion was based on seven different thresholds of GWAS significance ( $\mathrm{p}$-value thresholds $<0.01,0.05,0.10,0.20,0.30,0.40,0.50$ ) to determine the PRS that optimally contributed to the interaction model. The MDD-PRS was generated using PRS-on-Spark (35).

Population Stratification. To account for potential confounding by population structure within our sample (i.e. population stratification) $(36,37)$, we adjusted the outcome variables using the first two principal components of the genetic data (details in Supplementary Materials S2, and S3 Figure 1). The adjustment was performed using a multivariate linear mixed model, allowing for repeated measures on individuals and for correlations between distinct outcomes.

Adjusted Stress scores. Two adjusted scores were calculated. First, adjusted average distress scores (a mother's distress score averaged across time, Average Distress Model, ADM) and 
second, adjusted centered distress trajectory scores (a mother's distress score at a respective time point in relation to her average distress score, Centered Trajectory Model, CTM). A value >0 indicates that a mother has an adjusted score at the respective time point that is greater than her average score.

\section{Statistical Analyses}

Both models for ADM and CTM were fit using Latent Gold®, a software package for LC analysis developed by Statistical Innovations (Belmont, MA). In the Latent Gold® framework, we used the postnatal centered trajectory distress scores (after adjusting for population structure and centering) as the dependent response variables, the psychosocial factors and MDD-PRS as covariates (exogenous variables influencing the latent class that may vary between, but not within subjects), and months postpartum as predictors (exogenous variables that may vary within subjects). The ADM was modeled with class dependent constants, while the CTM was modeled with class dependent intercepts, slopes, and quadratic terms. We chose the regression submodule of Latent Gold®, which allows for the number of responses to vary across subjects. Generally, the latent class models used here followed the structure shown in Figure 1a). This structure implies that maternal genetics and psychosocial background together contribute to determining a latent susceptibility to distress. Models were specified with the following three steps: In step 1, we determined the number of latent classes for each of ADM and CTM models; in step 2, we selected the MDD-PRS at a p-value threshold yielding the greatest $\mathrm{R}^{2}$ in the given model and the SNPs of the best PRS was analyzed using the gene enrichment analysis tool of the MetacoreC 
software (Clarivate Analytics); in step 3, we examined the significance of the psychosocial covariates. The details of the respective steps are given in Figure $1 \mathrm{~b}$ ).

a)

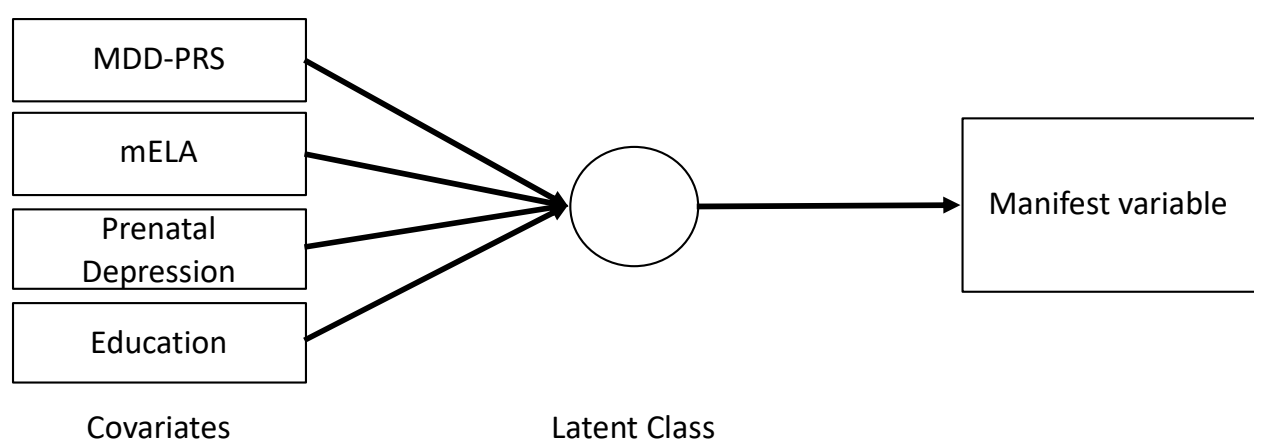

b)

Step 1: Number of latent classes

Selection of an appropriate number of latent classes for each model (ADM and CTM) by fitting models without covariates and varying the number of latent classes (1 to 10 classes). The number of latent classes that generated models with minimum Bayesian information criterion (BIC) values, or models falling in the bend when BIC was plotted against number of latent classes, was chosen as the appropriate number of latent classes (Supplementary Figures 2 and 5) .

\section{Step 2: MDD-PRS p-value threshold}

Examination of the interaction between MDD-PRS at a given $p$-value threshold for SNP inclusion and psychosocial covariates on class membership in each of the two models specified in step 1 . When a potentially interesting effect was identified in the interaction of MDD-PRS with a covariate ( $p$-value $<0.05$ ), we chose the MDDPRS with the threshold that produced the optimal model effect (i.e. the greatest Rsquared value, Supplementary Figure 4).

Step 3: Significant psychosocial covariates

Addition of the remaining psychosocial covariates to the latent class model with backward elimination. A p-value argument ( $p$-value $>0.05$ ) was applied for dropping a given covariate to obtain a final model.

Figure 1. a) General structure of the latent class models used in this study and b) Statistical procedure to specify the ADM and CTM latent class models. Abbreviations: ADM: Average Distress Model; BIC: Baye's Information Criterion; CTM: Centered Trajectory Model; MDDPRS: Polygenic Risk Score for Major Depressive Disorder; mELA: maternal history of early life adversities. 


\section{Results}

\section{Average Distress Models (ADM)}

A four-class solution yielded the minimum BIC for ADM LC models without covariates (step 1)

(See S3, Figure 2). The distributions of raw distress scores per class are shown in Supplementary Material S3, Figure 3. We identified a significant interaction between MDD-PRS and prenatal CES-D scores (step 2), with an MDD-PRS constructed at a $\mathrm{p}<0.1$ threshold exhibiting the largest effect (S3, Figure 4). After inclusion of this prenatal CES-D by MDD-PRS interaction term in the model, all other psychosocial covariates were significantly associated with class membership (step 3): MDD-PRS and prenatal CES-D score: Wald Interaction(3)=13.19, $p=0.004$; Wald MDD$\operatorname{PRS}(3)=6.02, p=0.11 ;$ Wald $_{\text {CES-D }}(3)=41.96, p<0.001$; Maternal Education: Wald Educ. $_{\text {(3) }}=11.61$, $p=0.009$; maternal mELA: WaldmELA(3)=8.64, $p=0.035$. After introducing the covariates, the characteristics of the classes were re-estimated (Figure 2).

Of the four classes, Class $1(n=52)$ had the highest values of maternal distress (Figure 2). The contour plot in Figure 3A illustrates the effect of the interaction: at a high MDD-PRS (i.e. high genetic risk for MDD), Class 1 membership probability increases with higher scores of CES-D; similarly, at a high score on CES-D, there is an increased membership probability as MDD-PRS increases. Hence Class 1 membership probability was highest for mothers with a high MDD-PRS and high prenatal CES-D scores. Additionally, mothers exposed to high mELA and without a university degree were more likely to belong to this class as compared to mothers with no mELA and with a university degree (Figure 4B). An enrichment analysis of genes included within the MDD-PRS revealed significant enrichment for a number of network processes 
including Gonadotropin Releasing Hormone Regulation (see Table 4), which is a system implicated in both reproduction and depression (38).

Levels of distress in Class 2 ( $n=97)$ were the second highest of the four classes (Figure 2). Again, mothers with high prenatal CES-D scores combined with a high MDD-PRS were more likely to belong to Class 2 than mothers with low prenatal CES-D and MDD-PRS (Figure 3B). In contrast to Class 1 , however, mothers holding a university degree were more likely to belong to Class 2 as compared to mothers without a university degree (Figure 4B), and membership probability was independent of mELA.

Class 3 ( $n=72)$ showed the second lowest levels of distress (Figure 2), and higher levels of mELA were associated with higher membership probability in this class (Figure 4A), suggesting that this was an "environmental risk"-only group. Distress levels in Class $4(n=40)$ were the lowest of all classes. In contrast to Class 1 and 2, mothers were more likely to belong to Class 4 when they had low levels of prenatal CES-D or low levels of MDD-PRS (Figure 3D) as well as low mELA (Figure 4A) but there was no association with maternal education. Detailed statistical information is provided in Table 2. 
Table 2

Four Class Average Distress Model (ADM) with Covariates

\begin{tabular}{|c|c|c|c|c|c|}
\hline Class & Term & Coefficient & $S E$ & $\bar{Z}$ & $p$ \\
\hline \multirow{7}{*}{ Class 1} & Intercept & -1.829 & 0.390 & -4.695 & $<.001$ \\
\hline & MDD-PRS $(\mathrm{p}<.1)^{1}$ & -0.413 & 0.395 & -1.045 & 0.300 \\
\hline & Prenatal CES-D Score & 0.187 & 0.029 & 6.429 & $<.001$ \\
\hline & Maternal Level of Education ${ }^{2}$ & -1.065 & 0.380 & -2.806 & 0.005 \\
\hline & $\mathrm{mELA}^{3}$ & 0.486 & 0.187 & 2.598 & 0.009 \\
\hline & MDD-PRS $(\mathrm{p}<.1) X$ Prenatal CES-D & & & & \\
\hline & Score & 0.064 & 0.030 & 2.134 & 0.033 \\
\hline \multirow[t]{7}{*}{ Class 2} & Intercept & -0.027 & 0.299 & -0.089 & 0.930 \\
\hline & MDD-PRS $(\mathrm{p}<.1)^{1}$ & -0.476 & 0.264 & -1.802 & 0.072 \\
\hline & Prenatal CES-D Score & 0.063 & 0.025 & 2.472 & 0.013 \\
\hline & Maternal Level of Education ${ }^{2}$ & 0.692 & 0.255 & 2.716 & 0.007 \\
\hline & mELA $^{3}$ & -0.016 & 0.184 & -0.085 & 0.930 \\
\hline & MDD-PRS ( $\mathrm{p}<.1)$ X Prenatal CES-D & & & & \\
\hline & Score & 0.089 & 0.026 & 3.409 & $<.001$ \\
\hline \multirow[t]{7}{*}{ Class 3} & Intercept & 1.082 & 0.357 & 3.031 & 0.002 \\
\hline & MDD-PRS $(\mathrm{p}<.1)^{1}$ & 0.185 & 0.314 & 0.590 & 0.560 \\
\hline & Prenatal CES-D Score & -0.051 & 0.031 & -1.621 & 0.100 \\
\hline & Maternal Level of Education ${ }^{2}$ & 0.223 & 0.300 & 0.743 & 0.460 \\
\hline & $\mathrm{mELA}^{3}$ & 0.376 & 0.189 & 1.985 & 0.047 \\
\hline & MDD-PRS $(\mathrm{p}<.1) X$ Prenatal CES-D & & & & \\
\hline & Score & 0.005 & 0.034 & 0.157 & 0.880 \\
\hline \multirow[t]{7}{*}{ Class 4} & Intercept & 0.774 & 0.458 & 1.690 & 0.091 \\
\hline & MDD-PRS $(\mathrm{p}<.1)^{1}$ & 0.703 & 0.338 & 2.080 & 0.038 \\
\hline & Prenatal CES-D Score & -0.199 & 0.052 & -3.817 & $<.001$ \\
\hline & Maternal Level of Education ${ }^{2}$ & 0.150 & 0.366 & 0.410 & 0.680 \\
\hline & mELA $^{3}$ & -0.846 & 0.396 & -2.134 & 0.033 \\
\hline & MDD-PRS $(\mathrm{p}<.1) X$ Prenatal CES-D & & & & \\
\hline & Score & -0.158 & 0.050 & -3.153 & 0.002 \\
\hline
\end{tabular}

${ }^{1}$ MDD-PRS was z-transformed; ${ }^{2} 0=$ no University Degree, $1=$ with University Degree; ${ }^{3}$ First Principal Component Score obtained from five measures on mELA (Childhood Trauma Questionnaire scores: Physical Neglect and Abuse, Emotional Neglect and Abuse, Sexual Abuse, and Parental Bonding Instrument score for Maternal Care). 


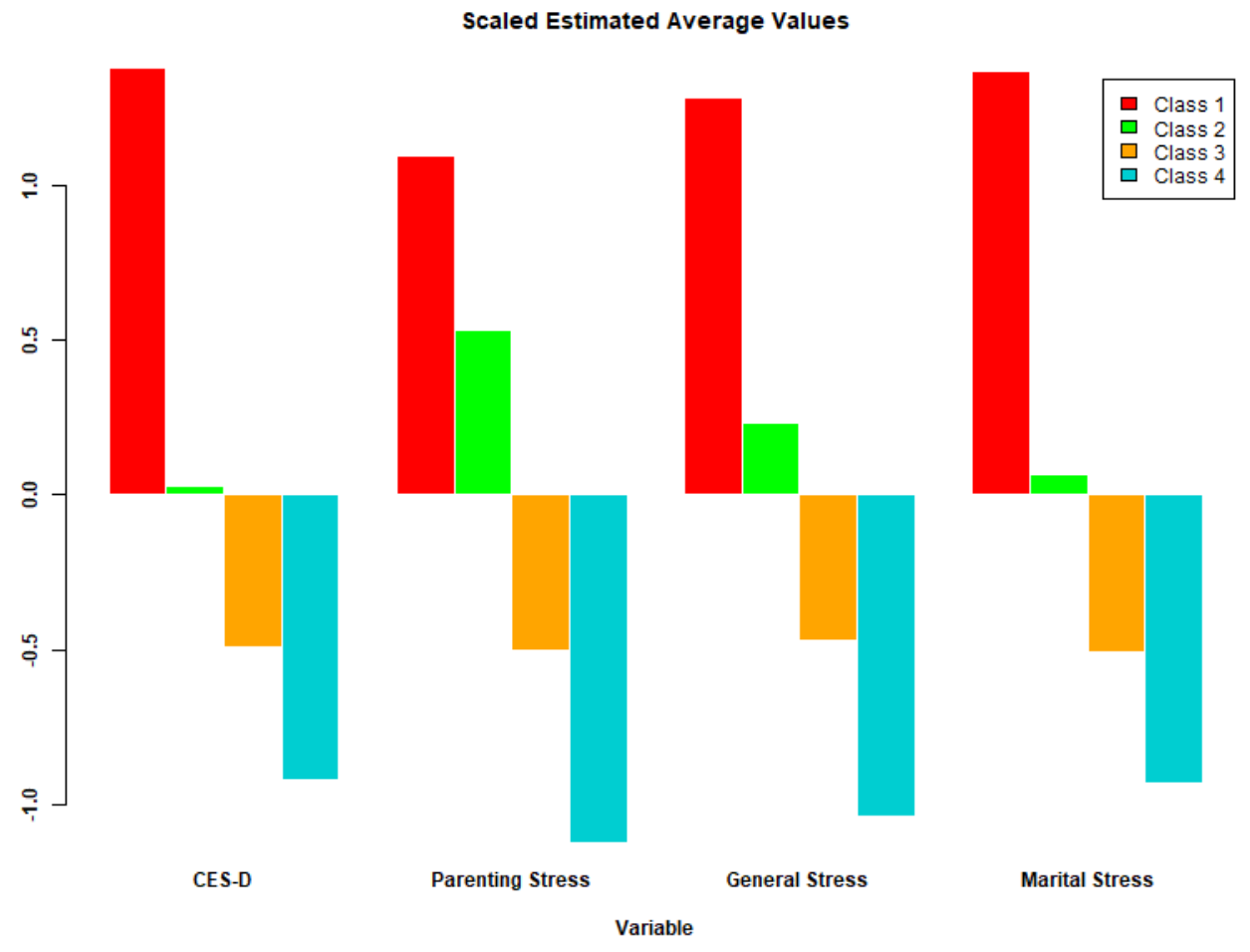

Figure 2. Estimated mean scores for symptoms of depression (CES-D), parenting stress (PSI), general stress (PSS) and marital stress (MSI) in the Average Distress Model (ADM). Mean scores were normalized using z-transformation $(M=0$ and $S D=1)$. Abbreviations: CES-D: Center of Epidemiological Studies Depression Score; MSI: Marital Stress Index; PSI: Parental Stress Index; PSS: Perceived Stress Scale. 

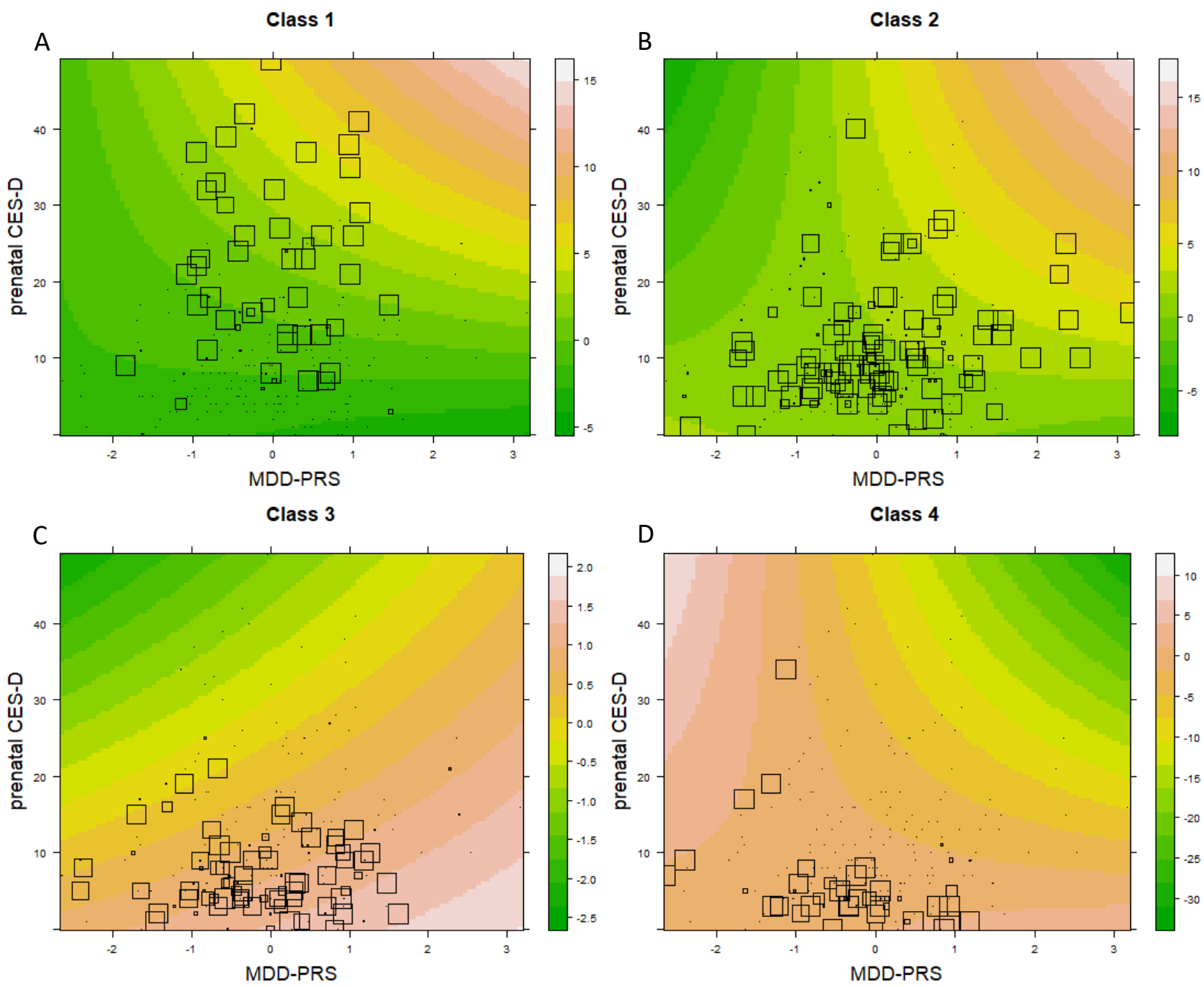

Figure 3. The contour plots show the ADM class membership probabilities (on the logit scale) of belonging to Class 1 (A), Class 2 (B), Class 3 (C) and Class 4(D) over differing values of prenatal CES-D and MDD-PRS, and with mother education level at a fixed value of 1 and mELA fixed at the average mELA value. The squares on the plots correspond to a subject (plotted at their value of MDD-PRS and prenatal CES-D). The size of the squares is proportional to the probability of belonging to the respective class, with larger squares indicating greater probability of class membership. Abbreviations: ADM: Average Distress Model; CES-D: Center of Epidemiological Studies Depression Score; mELA: maternal history of early life adversities; MDD-PRS: Polygenic Risk Score for Major Depressive Disorder. 

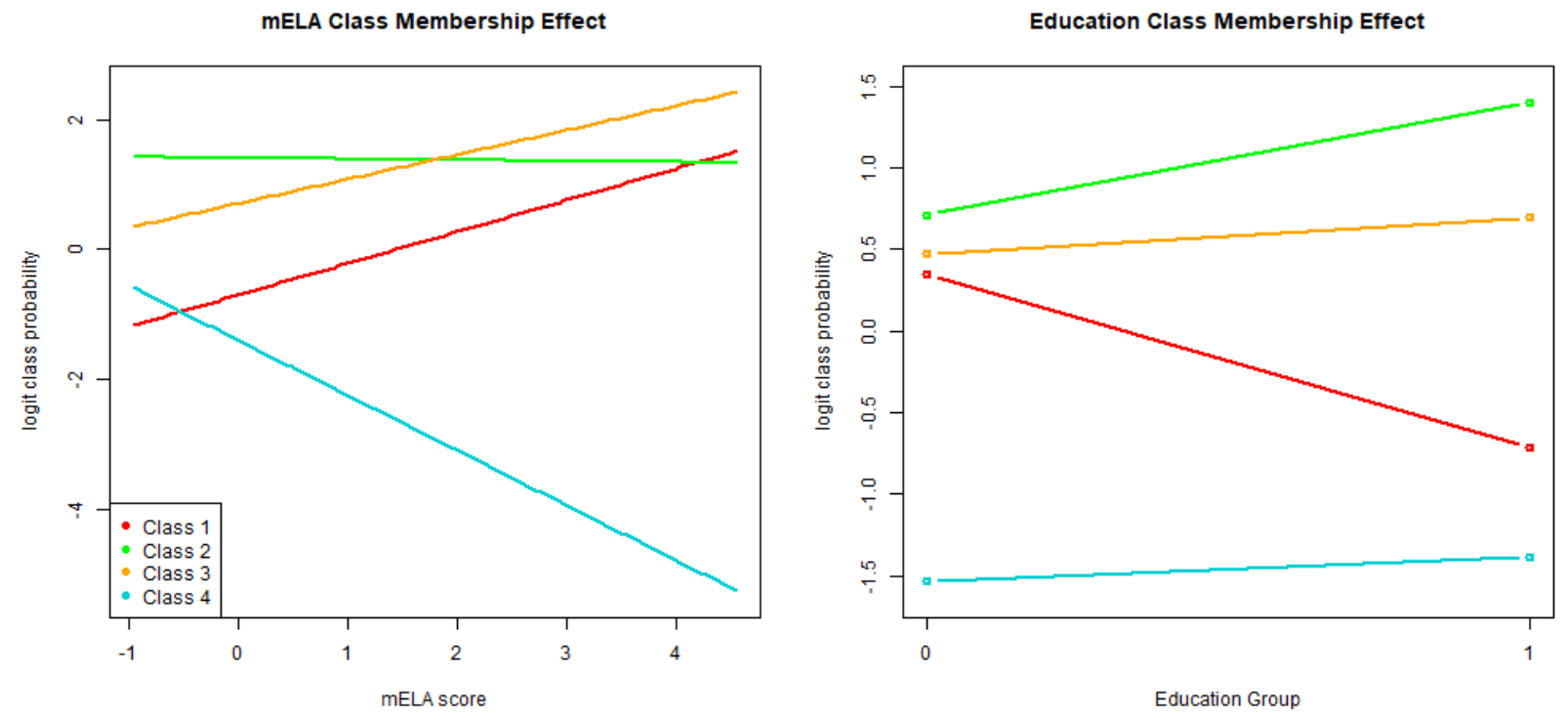

Figure 4. Association between covariates and average class membership probabilities for the Average Distress Model (ADM). A) association with mELA and B) maternal level of education $(0=$ no university degree; $1=$ with university degree $)$. Abbreviations: mELA: maternal history of early life adversities.

\section{Centered Trajectory Model (CTM)}

For trajectory scores centered across time, the BIC reached a minimum at seven classes. However, the more parsimonious and easier to interpret five-class solution yielded a BIC differing only slightly from the minimum (S3, Figure 5); therefore, the five-class solution was selected (step 1). The CTM did not show any evidence of a significant MDD-PRS by psychosocial covariate interaction (step 2), nor a main effect for MDD-PRS on class membership probability. Only mELA showed a significant main effect on the class probabilities of the CTM (WaldmELA $(3)=12.67, p=0.013)$. As shown in Table 3, increasing values of mELA were 
associated with a shift in class assignment from Class 5 to Class 3, but no significant changes in the probabilities of the remaining classes.

Class 1 ( $n=27)$ was characterized by steeply increasing curves of all measures, rising from lower to higher levels of distress during the observation time window (6 months to 6 years postpartum). Class 2 ( $n=63$ ) trajectories followed a quadratic trend over time: levels of distress increased from 6 months until 3 or 4 years, and then, except for marital stress, decreased towards the 6 month scores until the child reached 6 years of age (Figure 5). Class $3(n=28)$ consisted of mixed trajectory paths across the different stress measures with depressive symptom and general stress severity following similar quadratic trends, relatively stable levels of parenting stress, and quadratic marital stress trajectory distinct from the other measures (Figure 5). Mothers scoring higher in mELA were more likely to belong to this Class 3 (Figure 6). Class 4 ( $n=67$ ) trajectories were mostly stable over time with all scores showing only a slight decrease in the first 3 years postpartum (Figure 5). Finally, trajectories in depressive symptom severity and general stress of Class 5 ( $n=76)$ were relatively stable across time (Figure 5A and Figure 5C), while parenting and marital stress levels increased slightly over the six years postpartum (Figure $5 \mathrm{~B}$ and 5D). 
Table 3

Five Class Centered Trajectories Model (CTM) with mELA as Covariate

\begin{tabular}{llcccc}
\hline Class & Term & Coefficient & $\boldsymbol{S E}$ & $\boldsymbol{Z}$ & $\boldsymbol{p}$ \\
\hline Class 1 & Intercept & -0.469 & 0.217 & -2.164 & 0.030 \\
& mELA $^{1}$ & -0.003 & 0.202 & -0.013 & 0.990 \\
Class 2 & Intercept $^{1}$ & 0.350 & 0.162 & 2.156 & 0.031 \\
& mELA $^{1}$ & -0.005 & 0.162 & -0.032 & 0.970 \\
Class 3 & Intercept $^{1}$ & -0.659 & 0.234 & -2.815 & 0.005 \\
& mELA $^{1}$ & 0.478 & 0.147 & 3.263 & 0.001 \\
Class 4 & Intercept & 0.360 & 0.160 & 2.257 & 0.024 \\
& mELA $^{1}$ & -0.150 & 0.158 & -0.948 & 0.340 \\
Class 5 & Intercept $^{1}$ & 0.418 & 0.141 & 2.963 & 0.003 \\
& mELA $^{1}$ & -0.320 & 0.171 & -1.874 & 0.061 \\
\hline
\end{tabular}

${ }^{1}$ First Principal Component Score obtained from five measures on mELA (Childhood Trauma Questionnaire scores: Physical Neglect and Abuse, Emotional Neglect and Abuse, Sexual Abuse, and Parental Bonding Instrument score for Maternal Care)

Abbreviations: mELA: maternal history of early life adversity score; SE:

Standard Error 

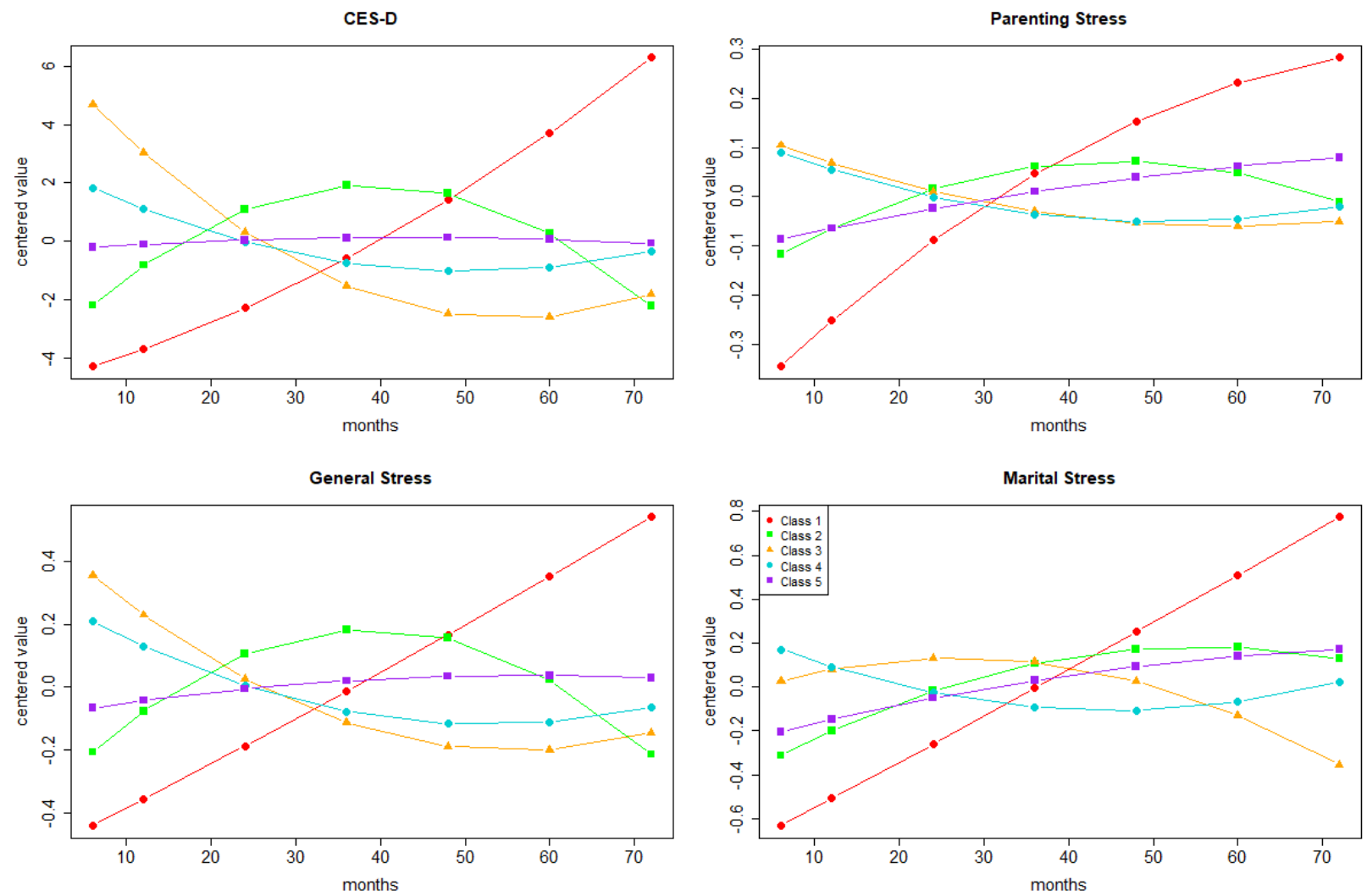

Figure 5. Class estimated values for the centered trajectory model (CTM) of A) symptoms of depression (CES-D); B) Parenting Stress (PSI); C) General Stress (PSS); and D) Marital Stress (MSI). Abbreviations: CES-D: Center of Epidemiological Studies Depression Score; MSI:

Marital Stress Index; PSI: Parental Stress Index; PSS: Perceived Stress Scale. 


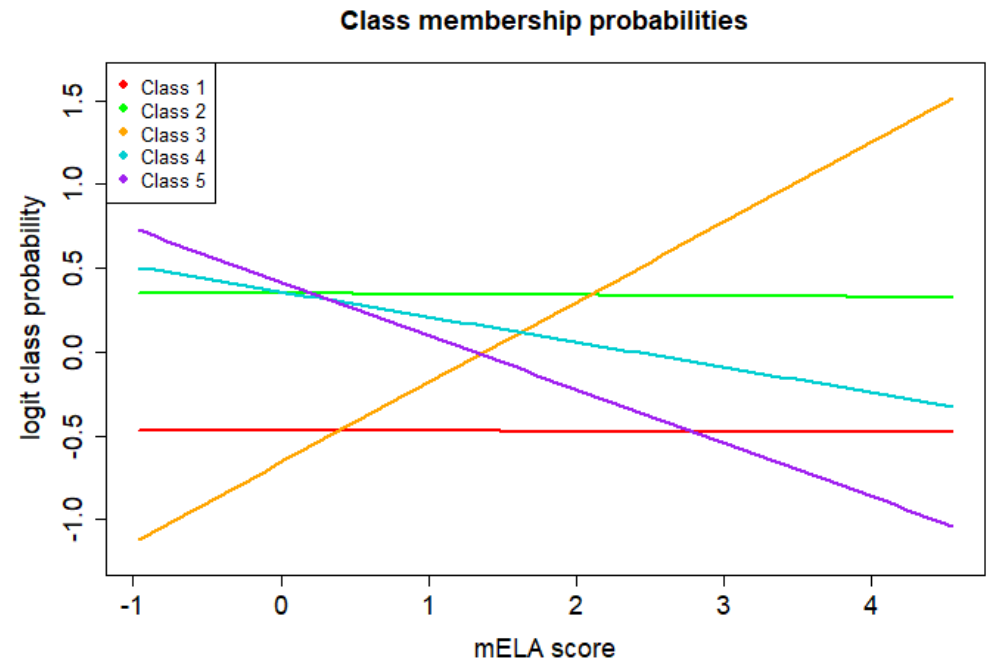

Figure 6. Association between mELA and centered class membership probabilities.

Abbreviations: mELA: maternal history of early life adversities. 
Table 4

Enrichment Analysis for Process Networks using the curated domains in Metacore

\begin{tabular}{lccccc}
\hline Term & In Data & Total & Ratio & p-value & FDR \\
\hline Neurophysiological process $\rightarrow$ Transmission of nerve impulse & 87 & 130 & $87 / 130$ & $4.2 \mathrm{E}-07$ & $6.7 \mathrm{E}-05$ \\
Cytoskeleton $\rightarrow$ Regulation of cytoskeleton rearrangement & 77 & 120 & $77 / 120$ & $2.1 \mathrm{E}-05$ & $1.7 \mathrm{E}-03$ \\
Development $\rightarrow$ Neurogenesis $\rightarrow$ Synaptogenesis & 77 & 122 & $77 / 122$ & $5.0 \mathrm{E}-05$ & $2.7 \mathrm{E}-03$ \\
Cell adhesion $\rightarrow$ Cell-matrix interactions & 91 & 150 & $91 / 150$ & $9.6 \mathrm{E}-05$ & $3.5 \mathrm{E}-03$ \\
Reproduction $\rightarrow$ Gonadotropin regulation & 69 & 109 & $69 / 109$ & $1.1 \mathrm{E}-04$ & $3.5 \mathrm{E}-03$ \\
Cell adhesion $\rightarrow$ Synaptic contact & 75 & 121 & $75 / 121$ & $1.5 \mathrm{E}-04$ & $4.0 \mathrm{E}-03$ \\
Reproduction $\rightarrow$ GnRH signaling pathway & 60 & 94 & $60 / 94$ & $2.2 \mathrm{E}-04$ & $4.8 \mathrm{E}-03$ \\
Cell adhesion $\rightarrow$ Integrin-mediated cell-matrix adhesion & 88 & 147 & $88 / 147$ & $2.4 \mathrm{E}-04$ & $4.8 \mathrm{E}-03$ \\
Neurophysiological process $\rightarrow$ GABAergic neurotransmission & 42 & 63 & $42 / 63$ & $5.1 \mathrm{E}-04$ & $9.0 \mathrm{E}-03$ \\
Transport $\rightarrow$ Synaptic vesicle exocytosis & 58 & 94 & $58 / 94$ & $9.9 \mathrm{E}-04$ & $1.6 \mathrm{E}-02$ \\
\hline Abbreval
\end{tabular}

Abbreviations: FDR: false discovery rate; GABA: gamma-Aminobutyric acid; GnRH: Gonadotropin releasing hormone 


\section{Discussion}

Applying a latent class approach for modeling multivariate trajectories in maternal distress including symptoms of depression, general daily life stress, parental-, and marital stress - during the first six years postpartum yielded four classes of mothers based on average distress (ADM). The probability of belonging to any of the four classes was affected by the interaction between maternal symptoms of prenatal depression and MDD-PRS, as well as mELA and level of education. Furthermore, we identified five classes based on the mothers' centered trajectories of distress (CTM), most of which were associated with mELA.

\section{Average Distress Model (ADM): mean levels of distress}

The scores of the four average distress classes were very consistent across all domains of distress (Figure 2), with Class 1 having the highest levels of symptoms of depression, parenting, marital, and general stress, Class 2 having the second highest levels, Class 3 the third highest and Class 4 the lowest levels. The effects of prenatal symptoms of depression, mELA, and maternal level of education are in line with previous findings linking these psychosocial risk factors to impaired wellbeing in the postpartum $(12,17-20,39,40)$. In our data, the PRS that best predicted levels of distress in the presence of psychosocial risk factors was enriched for genes involved in pathways related to neuronal functioning and reproduction. Elegant, functional experimental studies provide compelling evidence of individual differences in mood states associated with variation in hormonal mediators of reproductive (e.g., $\mathrm{GnRH})(38,41)$, which align well with our findings. An MDD-PRS by psychosocial environment interaction has been shown to predict MDD risk in 
adults $(21,22,42)$ but findings were mixed with regards to whether individuals with a high MDD-PRS were more or less vulnerable to psychosocial stress. A recent meta-analysis, however, found no significant interaction between MDD-PRS and childhood trauma in predicting MDD status (43). While a study on PRS in a sample of postpartum women found that postpartum depression was not associated with PRS-MDD, but with a PRS for bipolar disorder (23), this might be the first study addressing the interactions between psychosocial risk factors and MDD-PRS to predict postpartum wellbeing. Indeed, we found that the association of a higher MDD-PRS with postpartum distress depended on prenatal symptoms of depression, a more proximal measure as compared to mELA.

The probability of belonging to Class 1 - which had distress scores consistently higher than one standard deviation from the mean (Figure 2) - was increased for mothers with more severe mELA, without a university degree and with both high MDD-PRS and high prenatal symptoms of depression. This combination of risk factors suggests that these mothers were at risk for mood problems even before pregnancy. Class 2 was characterized by medium levels of depressive symptoms, general stress, and marital stress, while parenting stress was in the higher range (Figure 2). Mothers with high MDD-PRS and prenatal symptoms of depression were more likely to belong to Class 2, but compared to Class 1, membership probability was independent of mELA and was higher for mothers with a university degree. We speculate that a higher educational level might have diminished the effects of mELA and thus protected these mothers from clinically high distress scores observed in Class 1 (44). Maternal education, a marker of higher social class, might therefore buffer the effects of mELA (45). However, Class 2 showed relatively high levels of parenting stress, which might be due to reduced social support related to career choices demanding increased geographical mobility (46). Membership probability for 
Class 3, which showed distress scores approximately half a standard deviation below the mean, increased with severity of mELA, but was unrelated to all other factors investigated. Class 3 mothers might possess one or more biological or psychosocial factor (e.g. social support) protecting them from the adverse effects related to their childhood environment, independent of genetic risk for MDD, which remains to be identified. Finally, mothers were more likely to belong to Class 4 with the lowest distress levels (approximately one standard deviation below the mean) when they had a low genetic and/or a low psychosocial risk.

\section{Centered Trajectory Model (CTM): Change over time}

We identified five classes based on changes in distress after birth. In each class except Class 3 , the change over time in one measure was largely paralleled by similar changes in the other measures. Class 1 showed a very steep increase in all measures of postpartum distress. In Class 2, symptoms of depression and general stress increased until 3 years after birth and then returned to baseline levels, while parenting and marital stress peaked at around 4 years. We note that distress in mothers of Class 2 increased when the child gained more autonomy and that the recovery period probably coincided with the beginning of preschool. Class 3 trajectories showed slightly decreasing symptoms of depression and levels of general stress, and membership was increased with higher levels of mELA. This symptom profile observed in mothers assigned to Class 3 may reflect initial concerns about parental roles in individuals exposed to ELA (47). Class 4 showed only a slight decrease in distress, which was mostly limited to the early postpartum. Finally, Class 5 was characterized by very modest increases in all measures of distress and showed the greatest stability compared to the other classes. 
Two of the largest trajectory groups in this study (Class 4 and 5) were the ones showing the most stable levels of distress, representing almost $55 \%$ of our sample. This result agrees with previous research suggesting that depressive symptom severity is commonly stable in the postpartum $(6,20)$ and this stability may persist for years after giving birth $(48,49)$.

Finally, previous research suggests that mothers report higher relationship satisfaction during pregnancy with a decline postpartum $(50,51)$. This pattern was confirmed in our study, since mothers show a moderate (Classes 2 and 5, until 3 years postpartum Class 3 ) to strong (Class 1) increase in marital stress after birth. The reasons for increased marital stress might include unmet expectations of paternal involvement, or unbalanced childcare and household chores (52-54).

\section{Clinical Implications}

Our findings help identify relevant biological and environmental factors that contribute to individual differences in different domains of maternal distress over time. All of the factors investigated in this study could be assessed even during pregnancy to identify mothers with the highest risk for distress across the postpartum and to offer them additional support. Increased maternal wellbeing could in turn positively affect offspring psychosocial adjustment and neurodevelopment (55). Maternal postpartum distress impacts her personal and psychological parenting resources and is linked to decreased maternal sensitivity towards her child. Indeed, membership probability in the Class with the highest average distress levels (ADM Class 1), was associated to lower maternal sensitivity at 18 and 48 months, decreased school readiness of the child at 60 months, and higher levels of child-reported separation anxiety at 60 months (data not 
shown). Consequently, identifying mothers at risk for high levels of distress in the postpartum could support the planning of targeted interventions to prevent mothers and their children from developing psychosocial impairment.

\section{Strengths and Limitations}

We used an innovative approach to analyze longitudinal data and to exploit the multivariate assessment of maternal psychosocial stress in the postpartum. The decomposition of the trajectories into average distress and centered trajectory scores allowed us to address the change over time in postpartum distress specifically, which might be interesting in the clinical setting. Additionally, the data were derived from a longitudinal cohort study with multiple assessments in the extended postnatal period until school age, while many studies are restricted to the first three years after childbirth. Last, the inclusion of an interaction term between genetic risk and prenatal symptoms of depression provides information about the degree to which a mother suffered from prenatal symptoms of depression given her genetic risk.

Although promising, the results of this study need to be replicated in an independent cohort with analogous measures of distress, psychosocial, and genetic risk factors, to increase the generalizability of our findings. Moreover, as with many longitudinal studies, we had a high attrition rate due to study dropouts and missing data, resulting in a modest sample size, especially for a genetic study. Finally, we caution against interpreting the factors associated with membership probabilities as being causal. In particular, we did not examine any potentially stressful life events during the post-partum period, which might redirect the trajectory significantly to increased distress levels, thereby also affecting mean distress levels. 


\section{Conclusion}

We applied an innovative statistical approach accounting for the heterogeneity in the population to identify classes of mothers experiencing distinct longitudinal patterns of distress. Additionally, we assessed genetic and psychosocial factors associated with these classes. Our

findings could be relevant in a clinical setting, e.g. for identifying mothers at risk for high levels of distress in the postpartum and thus the planning of targeted interventions supporting mother and child mental wellbeing. 


\section{Acknowledgements}

This study was funded by a Canadian Institutes of Health Research (CIHR, http://www.cihrirsc.gc.ca/e/193.html) trajectory grant for the MAVAN study, and by the Ludmer Centre for Neuroinformatics and Mental Health. EU received funding from the Swiss National Science Foundation (P2BSP1_151913). The funders had no role in study design, data collection and analysis, decision to publish, or preparation of the manuscript. We like to thank all students and research assistants working in the MAVAN research team. We would also like to thank all the families participating in the MAVAN study.

A preliminary version of this data analysis was presented at the $25^{\text {th }}$ Annual Meeting of the International Genetic Epidemiology Society in Toronto, Canada, in 2016.

\section{Disclosures: none}




\section{References}

1. Barrett J, Fleming AS (2011): Annual Research Review: All mothers are not created equal: neural and psychobiological perspectives on mothering and the importance of individual differences. J Child Psychol Psychiatry. 52:368-397.

2. Fleming AS, Kraemer GW, Gonzalez A, Lovic V, Rees S, Melo A (2002): Mothering begets mothering: the transmission of behavior and its neurobiology across generations. Pharmacol Biochem Behav. 73:61-75.

3. Agrati D, Lonstein JS (2016): Affective changes during the postpartum period: Influences of genetic and experiential factors. Horm Behav. 77:141-152.

4. Vliegen N, Casalin S, Luyten P (2014): The course of postpartum depression: a review of longitudinal studies. Harv Rev Psychiatry. 22:1-22.

5. Meaney MJ (2018): Perinatal maternal depressive symptoms as an issue for population health. Am J Psychiatry.appiajp201817091031.

6. Santos H, Jr., Tan X, Salomon R (2016): Heterogeneity in perinatal depression: how far have we come? A systematic review. Arch Womens Ment Health.

7. Kinsella MT, Monk C (2009): Impact of maternal stress, depression and anxiety on fetal neurobehavioral development. Clin Obstet Gynecol. 52:425-440.

8. O'Connor TG, Monk C, Fitelson EM (2014): Practitioner review: maternal mood in pregnancy and child development--implications for child psychology and psychiatry. J Child Psychol Psychiatry. 55:99-111.

9. Crnic KA, Booth CL (1991): Mothers' and fathers' perceptions of daily hassles of parenting across early childhood. Journal of Marriage and the Family.1042-1050. 
10. Gelfand DM, Teti DM, Radin Fox CE (1992): Sources of parenting stress for depressed and nondepressed mothers of infants. Journal of Clinical Child and Adolescent Psychology. 21:262272.

11. Yim IS, Tanner Stapleton LR, Guardino CM, Hahn-Holbrook J, Dunkel Schetter C (2015): Biological and psychosocial predictors of postpartum depression: systematic review and call for integration. Annu Rev Clin Psychol. 11:99-137.

12. Beck CT (2001): Predictors of postpartum depression: an update. Nurs Res. 50:275-285.

13. Figueiredo FP, Parada AP, de Araujo LF, Silva WA, Jr., Del-Ben CM (2015): The Influence of genetic factors on peripartum depression: A systematic review. J Affect Disord. 172:265-273. 14. Rantalainen V, Binder EB, Lahti-Pulkkinen M, Czamara D, Laivuori H, Villa PM, et al. (2020): Polygenic prediction of the risk of perinatal depressive symptoms. Depress Anxiety. $37: 862-875$.

15. Wray NR, Lee SH, Mehta D, Vinkhuyzen AA, Dudbridge F, Middeldorp CM (2014): Research review: Polygenic methods and their application to psychiatric traits. $J$ Child Psychol Psychiatry. 55:1068-1087.

16. Major Depressive Disorder Working Group of the Psychiatric GC, Ripke S, Wray NR, Lewis CM, Hamilton SP, Weissman MM, et al. (2013): A mega-analysis of genome-wide association studies for major depressive disorder. Mol Psychiatry. 18:497-511.

17. Choi KW, Sikkema KJ (2016): Childhood maltreatment and perinatal mood and anxiety disorders: A systematic review. Trauma Violence Abuse. 17:427-453.

18. Jonas W, Mileva-Seitz V, Girard AW, Bisceglia R, Kennedy JL, Sokolowski M, et al. (2013): Genetic variation in oxytocin rs2740210 and early adversity associated with postpartum depression and breastfeeding duration. Genes Brain Behav. 12:681-694. 
19. Madigan S, Wade M, Plamondon A, Vaillancourt K, Jenkins JM, Shouldice M, et al. (2014): Course of depression and anxiety symptoms during the transition to parenthood for female adolescents with histories of victimization. Child Abuse Negl. 38:1160-1170.

20. McCall-Hosenfeld JS, Phiri K, Schaefer E, Zhu J, Kjerulff K (2016): Trajectories of depressive symptoms throughout the peri- and postpartum period: results from the First Baby Study. J Womens Health (Larchmt). 25:1112-1121.

21. Mullins N, Power RA, Fisher HL, Hanscombe KB, Euesden J, Iniesta R, et al. (2016): Polygenic interactions with environmental adversity in the aetiology of major depressive disorder. Psychol Med. 46:759-770.

22. Peyrot WJ, Milaneschi Y, Abdellaoui A, Sullivan PF, Hottenga JJ, Boomsma DI, et al. (2014): Effect of polygenic risk scores on depression in childhood trauma. Br J Psychiatry. 205:113-119.

23. Byrne EM, Carrillo-Roa T, Penninx BW, Sallis HM, Viktorin A, Chapman B, et al. (2014): Applying polygenic risk scores to postpartum depression. Arch Womens Ment Health. 17:519528.

24. O'Donnell KA, Gaudreau H, Colalillo S, Steiner M, Atkinson L, Moss E, et al. (2014): The maternal adversity, vulnerability and neurodevelopment project: theory and methodology. Can $J$ Psychiatry. 59:497-508.

25. Radloff LS (1977): The CES-D scale a self-report depression scale for research in the general population. Applied psychological measurement. 1:385-401.

26. Berry JO (1995): The Parental Stress Scale: Initial psychometric evidence. Journal of Social and Personal Relationships. 12:463-472.

27. Pearlin LI, Schooler C (1978): The structure of coping. J Health Soc Behav. 19:2-21. 
28. Cohen S, Kamarck T, Mermelstein R (1983): A global measure of perceived stress. J Health Soc Behav. 24:385-396.

29. Parker G, Tupling H, Brown LB (1979): Parental Bonding Instrument. British Journal of Medical Psychology. 52:1-10.

30. Bernstein DP, Ahluvalia T, Pogge D, Handelsman L (1997): Validity of the Childhood Trauma Questionnaire in an adolescent psychiatric population. J Am Acad Child Adolesc Psychiatry. 36:340-348.

31. Bouvette-Turcot AA, Fleming AS, Wazana A, Sokolowski MB, Gaudreau H, Gonzalez A, et al. (2015): Maternal childhood adversity and child temperament: an association moderated by child 5-HTTLPR genotype. Genes Brain Behav. 14:229-237.

32. Chang CC, Chow CC, Tellier LC, Vattikuti S, Purcell SM, Lee JJ (2015): Second-generation PLINK: rising to the challenge of larger and richer datasets. Gigascience. 4:7.

33. McCarthy S, Das S, Kretzschmar W, Delaneau O, Wood AR, Teumer A, et al. (2016): A reference panel of 64,976 haplotypes for genotype imputation. Nat Genet. 48:1279-1283.

34. Wray NR, Goddard ME, Visscher PM (2007): Prediction of individual genetic risk to disease from genome-wide association studies. Genome Res. 17:1520-1528.

35. Chen LM, Yao N, Garg E, Zhu Y, Nguyen TTT, Pokhvisneva I, et al. (2018): PRS-on-Spark (PRSoS): a novel, efficient and flexible approach for generating polygenic risk scores. $B M C$ Bioinformatics. 19:295.

36. Patterson N, Price AL, Reich D (2006): Population structure and eigenanalysis. PLoS Genet. 2:e190. 
37. Price AL, Patterson NJ, Plenge RM, Weinblatt ME, Shadick NA, Reich D (2006): Principal components analysis corrects for stratification in genome-wide association studies. Nat Genet. 38:904-909.

38. Mehta D, Rex-Haffner M, Sondergaard HB, Pinborg A, Binder EB, Frokjaer VG (2019): Evidence for oestrogen sensitivity in perinatal depression: pharmacological sex hormone manipulation study. Br J Psychiatry. 215:519-527.

39. Tebeka S, Le Strat Y, Dubertret C (2016): Developmental trajectories of pregnant and postpartum depression in an epidemiologic survey. J Affect Disord. 203:62-68.

40. Kim Y, Dee V (2018): Sociodemographic and obstetric factors related to symptoms of postpartum depression in hispanic women in rural California. J Obstet Gynecol Neonatal Nurs. 47:23-31.

41. Frokjaer VG, Pinborg A, Holst KK, Overgaard A, Henningsson S, Heede M, et al. (2015): Role of serotonin transporter changes in depressive responses to sex-steroid hormone manipulation: A positron emission tomography study. Biol Psychiatry. 78:534-543. 42. Colodro-Conde L, Couvy-Duchesne B, Zhu G, Coventry WL, Byrne EM, Gordon S, et al. (2017): A direct test of the diathesis-stress model for depression. Mol Psychiatry. 43. Peyrot WJ, Van der Auwera S, Milaneschi Y, Dolan CV, Madden PAF, Sullivan PF, et al. (2017): Does childhood trauma moderate polygenic risk for depression? A meta-analysis of 5765 subjects from the Psychiatric Genomics Consortium. Biol Psychiatry. 44. Montez JK, Hayward MD (2014): Cumulative childhood adversity, educational attainment, and active life expectancy among U.S. adults. Demography. 51:413-435. 
45. Mckenzie SK, Carter KN, Blakely T, Ivory V (2011): Effects of childhood socioeconomic position on subjective health and health behaviours in adulthood: how much is mediated by adult socioeconomic position? BMC Public Health. 11:269.

46. Parkes A, Sweeting H, Wight D (2015): Parenting stress and parent support among mothers with high and low education. J Fam Psychol. 29:907-918.

47. Agrati D, Browne D, Jonas W, Meaney M, Atkinson L, Steiner M, et al. (2015): Maternal anxiety from pregnancy to 2 years postpartum: transactional patterns of maternal early adversity and child temperament. Arch Womens Ment Health. 18:693-705.

48. Goodman JH (2004): Postpartum depression beyond the early postpartum period. J Obstet Gynecol Neonatal Nurs. 33:410-420.

49. Giallo R, Cooklin A, Nicholson JM (2014): Risk factors associated with trajectories of mothers' depressive symptoms across the early parenting period: an Australian population-based longitudinal study. Arch Womens Ment Health. 17:115-125.

50. Fleming AS, Ruble D, Krieger H, Wong PY (1997): Hormonal and experiential correlates of maternal responsiveness during pregnancy and the puerperium in human mothers. Horm Behav. $31: 145-158$.

51. Hjelmstedt A, Widstrom AM, Wramsby H, Collins A (2004): Emotional adaptation following successful in vitro fertilization. Fertil Steril. 81:1254-1264.

52. Craig L, Mullan K (2011): How mothers and fathers share childcare a cross-national time-use comparison. American Sociological Review. 76:834-861.

53. Ruble DN, Fleming AS, Hackel LS, Stangor C (1988): Changes in the marital relationship during the transition to first time motherhood: effects of violated expectations concerning division of household labor. J Pers Soc Psychol. 55:78-87. 
54. Cost KT, Jonas W, Unternaehrer E, Dudin A, Szatmari P, Gaudreau H, et al. (2018):

Maternal perceptions of paternal investment are associated with relationship satisfaction and breastfeeding duration in humans. J Fam Psychol. 32:1025.

55. Belsky J (1984): The determinants of parenting: a process model. Child Dev. 55:83-96. 